\title{
COMPLETELY REGULAR MAPS, FIBER MAPS AND LOCAL $n$-CONNECTIVITY
}

\author{
GERALD S. UNGAR
}

1. Introduction. It is well known that if $p$ is a Serre fibration from a space $E$ onto a space $B$ and $p^{-1}(b)$ is $n$-connected, then (1) $E$ is $n$-connected whenever $B$ is $n$-connected and (2) $B$ is $n+1$-connected whenever $E$ is $n+1$-connected. In this paper $n$-connected (or $n+1$ connected) is replaced by locally $n$ - (or $n+1-$ ) connected and similar theorems are proven. In addition, it is shown that Serre fibration can be replaced by completely regular map, and similar theorems (holding locally) can be obtained.

McAuley and Tulley [4] have proven the remaining result of this type on local $n$-connectedness for fiber maps. Specifically, if $p: E \rightarrow B$ is a Serre fibration from an $\mathrm{LC}^{n}$ metric space $E$ onto an $\mathrm{LC}^{n+1}$ metric space $B$, then not only are the fibers $\mathrm{LC}^{n}$ but $p$ is an $n$-regular map. In their paper Tulley proves a theorem which will be used several times in this paper. Since a modified statement of it is needed, it is quoted below.

(1.1) Theorem of Tulley. Suppose that $p: E \rightarrow B$ has the covering homotopy property for $n+1$-cells, $E$ and $B$ are metric spaces, and $E$ is LC ${ }^{0}$. Then small homotopies of $n$-cells can be lifted to small homotopies. (For the definitions, see [4].)

Dyer and Hamstrom [1] showed that completely regular mappings are locally trivial under certain conditions. Since fiber maps are a generalization of locally trivial maps, it is a natural question to ask if completely regular maps behave like fiber maps. It is shown in this paper that they do act almost like fiber maps with respect to local $n$-connectedness. However, it should be noted that in the hypothesis of (2.8) the degree of local connectedness is different from that needed for fiber maps.

The following notation will be used. An $\epsilon$-neighborhood of a point $b$ will be denoted by $N(b, \epsilon)$. If $f: X \rightarrow Y$ is a constant map $f(X)=y$, it will be denoted by $y^{*}$ (the domain will be obvious from the context). If $A_{1}$ and $A_{2}$ are contained in $A$ and $B_{1}$ and $B_{2}$ are contained in $B$, then $B^{A}\left(A_{1}, B_{1} ; A_{2}, B_{2}\right)$ will be the space of maps $\left\{f: A \rightarrow B \mid f\left(A_{1}\right)\right.$ $\subset B_{1}$ and $\left.f\left(A_{2}\right) \subset B_{2}\right\}$ with the compact open topology. If $A_{2}=B_{2}=\square$, the empty set, then $B^{A}\left(A_{1}, B_{1} ; A_{2}, B_{2}\right)$ will be denoted by $B^{A}\left(A_{1}, B_{1}\right)$.

Received by the editors February 1, 1968. 
2. Results. The following lemma will be used several times.

(2.1) Lemma. Let $B$ be a locally arcwise connected metric space. If $B^{I^{n}}$ (bdry $\left.I^{n}, b\right)$ is locally arcwise connected at $b^{*}$ for each $b$ in $B$, then $B$ is $n$-LC.

Proof. Let $b \in B$ and $\epsilon>0$ be given. Then there exists $\delta_{1}>0$ such that if $f:\left(I^{n}\right.$, bdry $\left.I^{n}\right) \rightarrow\left(N\left(b, \delta_{1}\right), b\right)$ then there exists an arc $\alpha$ in $B^{I^{n}}$ (bdry $I^{n}, b$ ) from $f$ to $b^{*}$, and the diameter of $\alpha$ is less than $\delta_{1}$. There also exists $\delta_{2}>0$ such that if $d(b, c)<\delta_{2}$ then $b$ and $c$ can be joined by an arc of diameter less than $\delta_{1}$.

Hence let $f:\left(I^{n}\right.$, bdry $\left.I^{n}\right) \rightarrow\left(N\left(b, \delta_{2}\right), c\right)$. Since $d(b, c)<\delta_{2}$ there exists an $\operatorname{arc} \alpha$ in $N\left(b, \delta_{1}\right)$ from $b$ to $c$. This induces [3, Theorem 14.1, p. 126] a map $g:\left(I^{n}\right.$, bdry $\left.I^{n}\right) \rightarrow\left(N\left(b, \delta_{2}\right), b\right)$ and hence there exists $G:\left(I^{n} \times I\right.$, (bdry $\left.\left.I^{n}\right) \times I\right) \rightarrow(N(b, \epsilon), b)$ such that $G_{0}=g$ and $G_{1}=b^{*}$. The path $\bar{\alpha}(t)=\alpha(1-t)$ acting on $G$ induces a map $H:\left(I^{n} \times I\right.$, (bdry $\left.\left.I^{n}\right) \times I\right) \rightarrow(N(b, \epsilon), c)$ such that $H_{0}$ is homotopic to $f$ in $N(b, \epsilon)$ and $H_{1}$ is homotopic to $c^{*}$ in $N(b, \epsilon)$. Hence it has been shown that given any $\epsilon>0$ and any $b \in B$, there exists $\delta>0$ such that if $f:\left(I^{n}\right.$, bdry $\left.I^{n}\right) \rightarrow(N(b, \delta), c)$ then there exists $F:\left(I^{n} \times I\right.$, (bdry $\left.\left.I^{n}\right) \times I\right)$ $\rightarrow(N(b, \epsilon), c)$ such that $F_{0}=f$ and $F_{1}=c^{*}$. It is easily seen that this condition implies that $B$ is $n$-LC.

(2.2) Note. If $p: E \rightarrow B$ has the covering homotopy property for $n$-cells then it has the covering homotopy property for $k$-cells $k \leqq n$.

(2.3) Theorem. Let $p: E \rightarrow B$ have the covering homotopy property for $n$-cells. Assume that $E$ and $B$ are metric spaces, $E$ is $\mathrm{LC}^{n}, p^{-1}(b)$ is $\mathrm{LC}^{n-1}$ for all $b$ in $B$ and $B$ is $\mathrm{LC}^{0}$. Then $B$ is $\mathrm{LC}^{n}$.

Proof. It will be shown that $B^{I^{k}}$ (bdry $\left.I^{k}, b\right)$ is locally arcwise connected at $b^{*}$ for every point $b$ in $B$ and for $k \leqq n$. Hence by (2.1) $B$ will be $\mathrm{LC}^{n}$.

Let $E_{k}=E^{I^{k}}\left(I^{k-1} \times 0, e ; I^{k-1} \times 1 \cup\left(\right.\right.$ bdry $\left.\left.I^{k}\right) \times I, p^{-1}(b)\right)$, where $e$ is some point in $p^{-1}(b)$. By [8] $E_{k}$ is LC 0 . Define $p_{*}: E_{k} \rightarrow B^{I^{k}}$ (bdry $\left.I^{k}, b\right)$ by $p_{*}(f)=p f$.

By the continuity of $p_{*}$, there exists $\delta_{1}>0$ such that if $d\left(f, e^{*}\right)<\delta_{1}$ then $d\left(p_{*}(f), p_{*}\left(e^{*}\right)\right)<\epsilon$. There also exists $\delta_{2}>0$ such that if $d\left(f, e^{*}\right)$ $<\delta_{2}$ then $f$ and $e^{*}$ can be joined by an arc of diameter less than $\delta_{1}$ in $E_{k}$. There also exists (by (1.1)) $\delta_{3}>0$ such that if $H: I^{k-1} \times I \rightarrow N\left(b, \delta_{3}\right)$ and $g: I^{k-1} \rightarrow N\left(e, \delta_{3}\right)$ are such that $p g(x)=H(x, 0)$, then there exists a lifting $G$ of $H$ relative to $g$ such that $G: I^{k-1} \times I \rightarrow N\left(e, \delta_{2}\right)$.

Therefore, let $\delta=\min \left\{\delta_{1}, \delta_{2}, \delta_{3}\right\}$ and let $f \in N\left(b^{*}, \delta\right) \subset B^{I^{k}}$ (bdry $\left.I^{k}, b\right)$. Then $f$ induces $H: I^{k-1} \times I \rightarrow N(b, \delta)$, and if $g: I^{k-1} \times 0 \rightarrow E$ is defined by $g(x, 0)=e$, then by the choice of $\delta_{3}$ there exists $G: I^{k-1} \times I \rightarrow N\left(e, \delta_{2}\right)$ 
such that $G_{0}=g, p G=H$, and $G\left(I^{k-1} \times 1 \cup\left(\right.\right.$ bdry $\left.\left.I^{k}\right) \times I\right) \subset p^{-1}(b)$. The map $G \in N\left(e^{*}, \delta_{2}\right)$ and hence can be joined by an arc of diameter less than $\delta_{1}$ to $e^{*}$. Therefore $p G=f$ is connected to $b^{*}$ by a path in $B^{I^{k}}\left(\right.$ bdry $\left.I^{k}, b\right)$ of diameter less than $\epsilon$. This completes the proof.

(2.4) Corollary. If $E, p$ and $B$ are as in (2.3) then $p$ is $n-1$ regular.

Proof. This follows from [4, Theorem 2].

(2.5) Corollary. If $p: E \rightarrow B$ is a completely regular closed map with compact fibers from a complete metric space $E$ onto a metric space $B$ and if $E$ is $L C^{n}, p^{-1}(b)$ is $\mathrm{LC}^{n}$ for each $b$ in $B$, and $B$ is $\mathrm{LC}^{0}$, then $B$ is $\mathrm{LC}^{n}$.

Proof. It is easily seen that $p$ is $n$-regular and hence $\left\{p^{-1}(b) \mid b \in B\right\}$ is an equi-LC ${ }^{n}$ collection. By [6, Theorem 3.4], $p$ has the covering homotopy property for $n$-cells. Therefore by (2.3) $B$ is $\mathrm{LC}^{n}$.

(2.6) Theorem. Let $p: E \rightarrow B$ have the covering homotopy property for $n+1$-cells. Assume that $E$ and $B$ are metric, $E$ is $\mathrm{LC}^{0}, B$ is $\mathrm{LC}^{n}$ and $p^{-1}(b)$ is $\mathrm{LC}^{n}$ for each $b$ in $B$. Then $E$ is $\mathrm{LC}^{n}$.

Proof. Let $e \in E$ and $k \leqq n$. By (2.1) it suffices to prove that $E^{I^{k}}$ (bdry $\left.I^{k}, e\right)$ is LC ${ }^{0}$. Let $\epsilon>0$ be given. Then, since $p^{-1} p(e)$ is $\mathrm{LC}^{n}$, there exists $\delta_{1}>0$ such that if $f:\left(I^{k}\right.$, bdry $\left.I^{k}\right) \rightarrow\left(p^{-1} p(e) \cap N\left(e, \delta_{1}\right), e\right)$ then there exists $F:\left(I^{k} \times I\right.$, (bdry $\left.\left.I^{k}\right) \times I\right) \rightarrow\left(p^{-1} p(e) \cap N(e, \epsilon), e\right)$ such that $F_{0}=f$ and $F_{1}=e^{*}$. By (1.1) there exists $\delta_{2}>0$ such that if $f: I^{k} \rightarrow N\left(e, \delta_{2}\right)$ and $G: I^{k} \times I \rightarrow N\left(p(e), \delta_{2}\right)$, and $p f(x)=G(x, 0)$, then there exists $H: I^{k} \times I \rightarrow N\left(e, \delta_{1}\right)$ satisfying $H(x, 0)=f(x)$ and $p H=G$. Since $B$ is $\mathrm{LC}^{n}$, there also exists $\delta_{3}>0$ such that if $f:\left(I^{k}\right.$, bdry $\left.I^{k}\right)$ $\rightarrow\left(N\left(p(e), \delta_{3}\right), p(e)\right)$ then $f$ could be extended to $F:\left(I^{k} \times I\right.$, bdry $\left.I^{k} \times I\right)$ $\rightarrow\left(N\left(p(e), \delta_{2}\right), p(e)\right)$ such that $F_{1}=p(e)^{*}$. Finally, by the continuity of $p$, there exists $\delta_{4}>0$ such that $p\left(N\left(e, \delta_{4}\right)\right) \subset N\left(b, \delta_{3}\right)$. It is easily seen that $\delta=\min \left\{\delta_{1}, \delta_{2}, \delta_{3}, \delta_{4}\right\}$ is the desired $\delta$ for the $k$-dimensional local connectedness at $e$.

(2.7) Corollary. If $E, p$ and $B$ are as in (2.6), then $p$ is $n-1$ regular.

Proof. This follows from [4, Theorem 2].

(2.8) Corollary. If $p: E \rightarrow B$ is a closed completely regular map with compact fibers from a complete metric space $E$ onto a metric space $B$ and if $E$ is $\mathrm{LC}^{0}, B$ is $\mathrm{LC}^{n}$, and $p^{-1}(b)$ is $\mathrm{LC}^{n+1}$ for each $b$ in $B$, then $E$ is $\mathrm{LC}^{n}$. 
Proof. It is easily seen that $p$ is $n+1$-regular and hence $\left\{p^{-1}(b) \mid b \in B\right\}$ is an equi-LC ${ }^{n+1}$ collection. By [6, Theorem 3.4] $p$ has the covering homotopy property for $n+1$-cells. Therefore by (2.6) $E$ is $\mathrm{LC}^{n}$.

(2.9) Corollary. Suppose that $p: E \rightarrow B$ is a Serre fibration from a complete metric space $E$ onto a finite-dimensional metric space $B$ such that each inverse under $f$ is homeomorphic to the compact 3-manifold with boundary $M$ and that either the Poincaré conjecture is true or each homotopy cell in $M$ is a 3-cell. Further, suppose that either

(a) $E$ is $\mathrm{LC}^{3}$ and $B$ is $\mathrm{LC}^{0}$ or

(b) $B$ is $\mathrm{LC}^{3}$ and $E$ is $\mathrm{LC}^{0}$.

Then $p$ is locally trivial.

Proof. By (2.3) or (2.6) $p$ is 2-regular and hence by [2] $p$ is locally trivial.

(2.10) Corollary. If $p: E \rightarrow B$ is Serre fibration from $E$ to $B$ and $\operatorname{dim} B \leqq n+1$ and

(a) $E$ is $\mathrm{LC}^{n}$ and $B$ is $\mathrm{LC}^{n+1}$ or

(b) $E$ is $\mathrm{LC}^{n+1}$ and $p^{-1}(b)$ is $\mathrm{LC}^{n}$ for all $b \in B$ or

(c) $B$ is $\mathrm{LC}^{n+1}$ and $p^{-1}(b)$ is $\mathrm{LC}^{n+1}$ for all $b \in B$, then $p$ admits local sections.

Proof. Since $a, b$ and $c$ imply $p$ is $n$-regular the conclusion follows from [5, Theorem 1.2].

Let $H(X)$ denote the space of homeomorphisms of $X$ onto itself with the compact open topology. If $x \in X$, let $H(X, x)$ be $\{h \in H(X) \mid h(x)=x\}$.

(2.11) CoRollary. If $X$ is a locally compact $\mathrm{LC}^{n}$ metric space such that $T_{x}: H(X) \rightarrow X$ defined by $T_{x}(h)=h(x)$ is a Serre fibration, then

(a) $H(X)$ is $\mathrm{LC}^{n}$ if $H(X, x)$ is $\mathrm{LC}^{n}$ and

(b) $H(X, x)$ is $\mathrm{LC}^{n-1}$ if $H(X)$ is $\mathrm{LC}^{n-1}$.

Proof. (a) follows from (2.6) and (b) follows from [4].

(2.12) Note. If $X$ is locally homogeneous in the sense of $\mathrm{Hu}$, then $X$ satisfies the hypothesis of (2.11) (see [7]).

\section{BIBLIOGRAPHY}

1. E. Dyer and M. E. Hamstrom, Completely regular mappings, Fund. Math. 45 (1957), 103-118.

2. M. E. Hamstrom, Regular mappings and the space of homeomorphism on a 3manifold, Mem. Amer. Math. Soc. No. 40 (1961), 1-42.

3. S. T. Hu, Homotopy theory, Academic Press, New York, 1959. 
4. L. McAuley and P. Tulley, Topology seminar, Wisconsin, 1965, edited by R. Bing and R. Bean, Ann. of Math. Studies, No. 60, Princeton Univ. Press, Princeton, N. J., 1966.

5. E. Michael, Continuous selections. II, Ann. of Math. (2) 64 (1956), 567-580.

6. - Continuous selections. III, Ann. of Math. (2) 65 (1957), 375-390.

7. G. Ungar, Local homogeneity, Duke Math. J. 34 (1967), 693-700.

8. H. Wada, Local connectivity of mappings spaces, Duke Math. J. 22 (1955), 419-425.

Louisiana State University 